\title{
Indoor Localization of Quadcopters in Industrial Environment
}

\author{
Péter TROLL ${ }^{\mathrm{a}, 1}$, Károly SZIPKA ${ }^{\mathrm{a}}$, Andreas ARCHENTI ${ }^{\mathrm{a}}$ \\ ${ }^{a}$ KTH Royal Institute of Technology, Department Production Engineering, \\ Brinellvägen 68, 10044 Stockholm, Sweden
}

\begin{abstract}
The research work in this paper was carried out to reach advanced positioning capabilities of unmanned aerial vehicles (UAVs) for indoor applications. The paper includes the design of a quadcopter and the implementation of a control system with the capability to position the quadcopter indoor using onboard visual pose estimation system, without the help of GPS. The project also covered the design and implementation of quadcopter hardware and the control software. The developed hardware enables the quadcopter to raise at least $0.5 \mathrm{~kg}$ additional payload. The system was developed on a Raspberry single-board computer in combination with a PixHawk flight controller. OpenCV library was used to implement the necessary computer vision. The Open-source software-based solution was developed in the Robotic Operating System (ROS) environment, which performs sensor reading and communication with the flight controller while recording data about its operation and transmits those to the user interface. For the vision-based position estimation, pre-positioned printed markers were used. The markers were generated by ArUco coding, which exactly defines the current position and orientation of the quadcopter, with the help of computer vision. The resulting data was processed in the ROS environment. LiDAR with Hector SLAM algorithm was used to map the objects around the quadcopter. The project also deals with the necessary camera calibration. The fusion of signals from the camera and from the IMU (Inertial Measurement Unit) was achieved by using Extended Kalman Filter (EKF). The evaluation of the completed positioning system was performed with an OptiTrack optical-based external multi-camera measurement system. The introduced evaluation method has enough precision to be used to investigate the enhancement of positioning performance of quadcopters, as well as fine-tuning the parameters of the used controller and filtering approach. The payload capacity allows autonomous material handling indoors. Based on the experiments, the system has an accurate positioning system to be suitable for industrial application.
\end{abstract}

Keywords. Quadcopter, Sensor fusion, Indoor localization, Fiducial marker

\footnotetext{
${ }^{1}$ Corresponding Author. ptroll@kth.se
} 


\section{Introduction}

An indoor localization system has the potential to affect application capabilities in the same way as satellite systems do outdoors. Indoor positioning is a challenging problem in a particularly complex and dynamic environment. There are several different approaches to solving the problem of indoor positioning, but there is currently no standardized solution to meet the needs of positioning in an indoor environment. It is known that position tracking systems already in use in factories and warehouses can greatly increase productivity by optimizing the use of resources. [1]

Based on the latest "Drone Industry Barometer 2019" from Drone Industry Insights [2] there is a definite need for robust and reliable UAV (Unmanned Aerial Vehicle) related algorithms and end-to-end solutions that provide solutions to key applications. Based on the report four most common industrial application examples can be defined: Mapping, Inspection / Maintenance, Surveying and Monitoring. Several other promising application areas can be identified, such as: Quality control, Remote maintenance, Measuring large-scale geometric shapes and volumes, Optical digitalization and scanning (both layout and parts), Logistics (an alternative to unmanned ground vehicles), Warehouse management, Collaborative applications, "3Ds" (dirty, dangerous and difficult) tasks. These applications could support the digitalisation and automatisation of industrial processes and reducing the use of resources. Several enabling technologies were explored in case of these applications. An important overlap between them was identified, which requires the accurate indoor positioning of UAVs under various loaded and unloaded conditions. To understand limitations and aim to push the boundaries of the state-of-the-art a research project was set up to address the positioning capabilities of UAVs under industrial environment.

\section{Background}

Multicopter's autonomic control system requires accurate real-time data about the actual position and orientation of the device. In open spaces reliable operation can be reached with the support of GPS technology if the system has sufficient satellite signal. However, for indoor use, where GPS signals are weak or inexistent, the use of another local positioning system becomes necessary. Furthermore, indoor applications can require a positioning accuracy, which are several orders of magnitude higher, then for open spaces. Industrial applications require robust solutions for accurate positioning, which can be only realized through multi-physics-based information and control. This introduces significant complexity in the design and testing of UAVs. At the same time, according to the state-of-the-art, it is not straight forward how the positioning performance of UAVs should be evaluated.

Some outdoor sensors also perform well indoors. For example, optical vision with an RGB-D camera, binocular stereo camera or OpticalFlow sensors are used to capture the 3D spatial information, to identify object, avoid obstacles or build a map. Laser rangefinder, LIDAR (Light detection and ranging) or Ultrasonic sensor can be used with Simultaneous Localization and Mapping (SLAM) algorithms to achieve autonomous navigation. The combination of the different sensors can support a robust system. 


\subsection{State-of-the-art of indoor localization}

Among applications that support indoor navigation, the fusion of separate sensors is common. The short-term drift accumulation of inertial navigation can be corrected by various external sensor sources. There are solutions where marker-based computer vision helps with precise landing. [3] Indoor localization technologies based on the physical approach can be divided into the following 3 main groups, according to [4]:

\subsubsection{Wave characteristics and propagation}

Ultrasound / ultrasonic positioning systems at room level have middle range accuracy and performance [5], but implementing on a large scale will decrease the effectiveness. Furthermore, ultrasound systems are very sensitive to noise and interference. Radio frequency-based positioning systems, for example, Bluetooth or WLAN or NFC-based positioning systems are very similarly unreliable for accurate indoor application. During operation the radio waves are disturbed by the absorption, reflection or interference of the surrounding objects[6]. Radio frequency-based technologies are only recommended for estimation of large-scale, less accurate positioning.

\subsubsection{Image /optical-based localization}

Among the fiducial marker-based positioning solutions, the two most widely used are ArUco and AprilTag markers [7]. This technology is a map-based system, where the position of the markers is predefined [8]. Generally, increasing the camera resolution reduces the positioning error, but increases the computational demand. Studies have shown that ArUco outperforms AprilTags in accuracy [9]. Although such a system can significantly reduce infrastructure costs, there are a number of challenges, such as motion blur and high-performance requirements for onboard image processing.

MotionCapture is an infrared marker-based indoor application that works with an external camera system, a map-based system that provides accurate absolute measurement in the area surrounded by cameras, but with significant infrastructure costs.

Laser-based systems like LiDARs provide more reliable accuracy [10] but are sensitive to reflection and absorption [11]. Using a SLAM algorithm, LiDAR can support a map-building system.

Optical Flow is a mapless system that measures the relative motion of the environment. It is sensitive for light conditions and the relative movements of the objects in the field of view of the sensor.

\subsubsection{Inertial navigation systems}

The microelectromechanical-system-based accelerometers, gyroscopes, and magnetometers provide motion and orientation data. The accelerometer detects the relative motion of the device in the form of acceleration. Their great advantage is the low power consumption, the small physical size, they do not require any external device or marker. The acceleration is double-integrated to determine the travelled distance, however, the measurement noise accumulates and causes an increasing positioning error over a period, this leads to poor accuracy in long term positioning, as well as changes in ambient temperature affect the drift and so the measurement accuracy. With a multi-sensor system and a well tuned filter algorithm like Kalman Filter or Particle Filter the drift can be tracked and compensated within certain limits and the short term stability can be extended. 


\section{Development of indoor localisation solution for UAVs}

Recent developments of unmanned aerial vehicles (UAVs) provide important opportunities for applications in industrial or hazardous environment. UAVs can be important tools in the future to reach higher levels of automation in tasks related to industrial metrology and inspection, logistics, or for instance production planning.

However, several technologies have to be integrated and some have to be developed in order to reach high technology readiness level applications.

This project deals with a quadcopter design with an onboard computer-assisted image processing with camera and LiDAR system combined with the onboard IMU. Data fusion is realized with the application of an Extended Kalman Filter in order to increase positioning performance. The focus is on the possible improvement of the overall accuracy of the positioning without the use of a GPS module.

\subsection{Hardware design}

A DJI 450 frame was used as hardware fame, which has the necessary requirements as a lifting power for onboard computer and sensors and additional payload and has the appropriate geometry for the above. The hardware design follows the traditional quadcopter design, as it is illustrated in Figure 1 below.

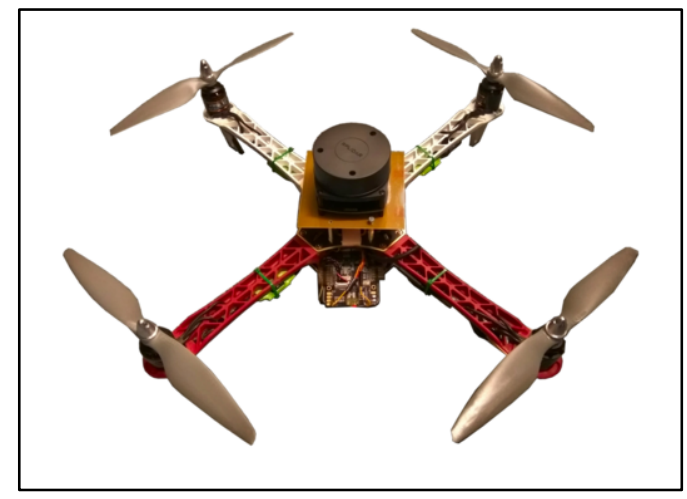

Figure 1. The assembled hardware of the UAV.

The onboard computer is a Raspberry Pi $3+$ model that communicates via serial connection with the PixHawk 4 Flight controller, the onboard sensors, in addition to the PixHawk Integrated Inertial Measurement Unit, a Raspberry Pi V2 Camera and an RPlidar A1.

For security reasons, communication with the device is redundant. In addition to the PC that sends commands over the WiFi network to the onboard computer, an additional external radio remote controller is integrated. This solution is important, in case the user needs to take over the control manually and immediately. The complete hardware design of the system is shown in the following Figure 2. 


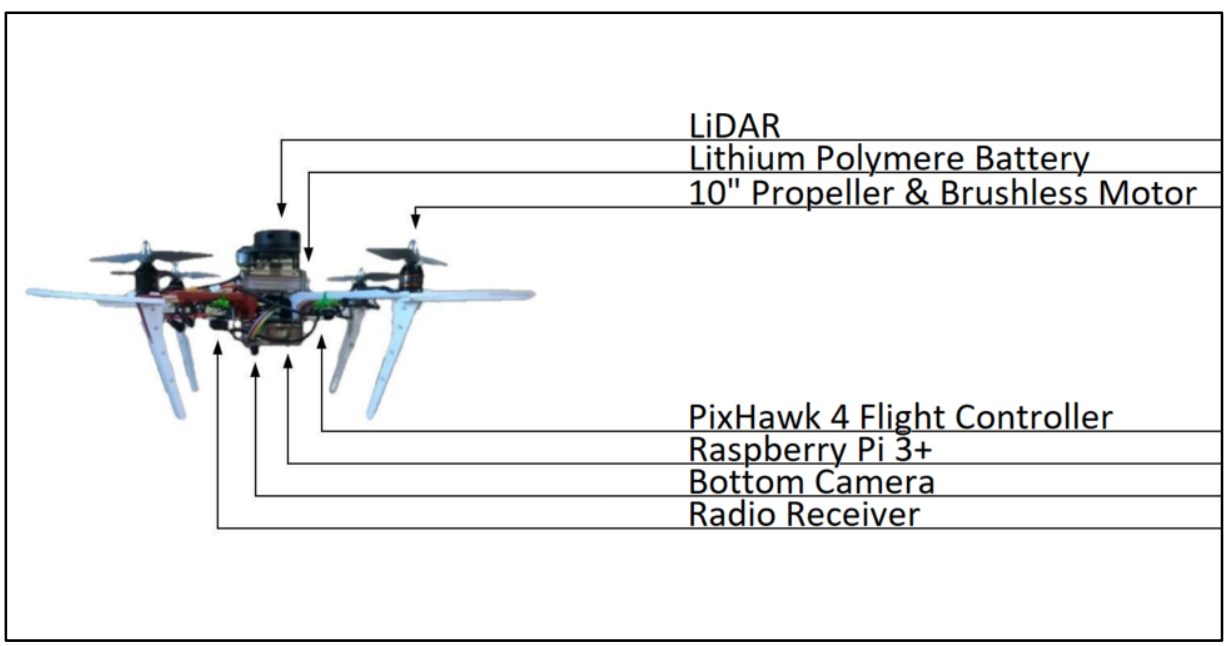

Figure 2. The main components of the hardware.

\subsection{Software design}

The system was implemented in ROS (Robot Operating System) environment. ROS has the ability to connect pieces of software called nodes together. Nodes can take care of tasks, for example, reading sensor data, if the node has information to publish, it shares the information using a topic. Other nodes can subscribe to the topics and read and use the shared information.

In the ROS environment, there are 5 different major units: MavROS, Waypoint navigation, external radio signal reading, OpenCV for the Computer Vision, and Hector SLAM [12], for the LiDAR-based SLAM algorithm as seen in Figure 3.

Furthermore, the ROS environment is responsible for the communication with the Flight controller and the Ground control station. The continuous connection to the quadcopter is realized by a PC connected via a WiFi network. Command-line communication on Linux enables real-time monitoring and control of the ROS environment. The data is visualized by the RViz tool which is the $3 \mathrm{D}$ visualization interface of the ROS environment.

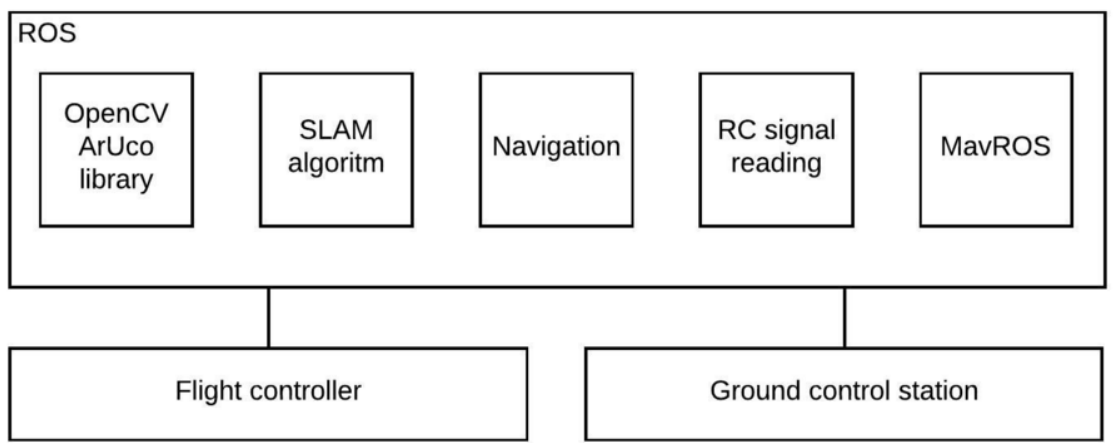

Figure 3. The main units of the software architecture. 
MAVROS is the main communication node for ROS. MAVROS can translate ROS topic messages and MAVLink messages. It allows the ROS on the Raspberry to communicate with the Pixhawk flight controller using its serial port. Additional it has UDP MAVLink bridge to communicate with the ground control station wirelessly.

MAVLink stands for Micro Aerial Vehicle Link is a standardized communication protocol for sending information. It allows bi-directional communication between the onboard subsystems of the quadcopter and the ground control station. The MAVLink protocol follows the publish-subscribe design pattern. MAVLink's benefits include being highly effective, reliable, supports many programming languages.

\subsection{Computer vision}

The Raspberry Pi V2 camera with 8-megapixel resolution and a wide-angle vision, compared to other raspberry pi compatible cameras it proves to be a good option for this application. However, due to the distortion of the lens of the camera, first it is necessary to calibrate the image of the camera so that the complete angle of vision of the mapped image is size-correct.

The camera has been calibrated using the traditional chessboard method, which has produced distortion coefficient and camera matrix that allows OpenCV functions to take advantage of the full field of view. After calibration, the connection between the camera's pixel units and the captured distances in the environment the relative position can be directly calculated.

For pose estimation, the OpenCV environment supports a marker-based position estimation in Cartesian coordinate system. The position estimation works with ArUco Markers [7] which is a popular solution for highly reliable position estimation, it uses binary square markers, the benefit of this markers is that only one marker in the Field of View is enough to determine the position and orientation of the camera relative to the marker.

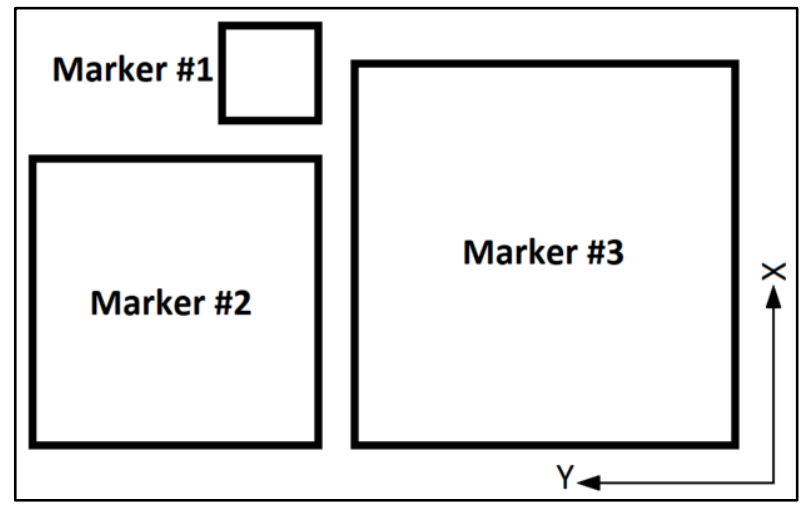

Figure 4. The introduced marker layout.

An example of the used markers can be seen in figure 4. The variation of the sizes supports better image resolution from different distances between the camera and the markers. The used marker sizes, in this case, are the followings $\# 1$ : 50x50mm, \#2: 150x150mm, \#3: 200x200mm. During take-off or landing, the smallest marker is in 
the field of view of the camera, and when it reaches the operating altitude, larger markers provide more reliable accuracy. In the case of simultaneous presence of multiple markers, the position data obtained from the markers is determined by averaging, however, this can be modified, for example, with a weighted estimation. It is crucial for the reliability of the marker-based positioning that at least one marker is in the sight of the camera and the patterns of the markers can be captured with a given camera resolution. The closely placed markers with various sizes assure reliable identification of markers and in many cases assures redundant information by simultaneously tracking more markers.

To integrate the ArUco module into the ROS environment the ROS ArUco package was used. This makes it possible to use more than one marker simultaneously to assist the position estimation. Ideally, markers shall be placed on a grid table, defining the sections of a common coordinate system. Within the ROS environment, the ArUco Node subscribing to the Camera topic compensates the distortion based on the calibration file. Markers are detected on the image and their position is identified within the coordinate system, and then publishes the pose data of the camera. The MavROS vision pose topic gets the camera pose message, then the MavROS generates ROS messages and sends it through MAVLink messages to the flight controller.

\subsection{SLAM}

In support of non-GPS-based positioning, the indoor environment can be monitored using a LIDAR system. In order to use such a sensor for localization, an algorithm must process the incoming signal to handle the change of the position. A widely used solution is SLAM (Simultaneous Localization And Mapping). SLAM is a technique for creating a map of environment and determining the device position at the same time. SLAM algorithms are able to merge the measurements from previous positions and the new, changed measurements. To use SLAM in the ROS environment, the Hector SLAM ROS package has been implemented. [12] the map generated by the algorithm is shown on the Figure 5.

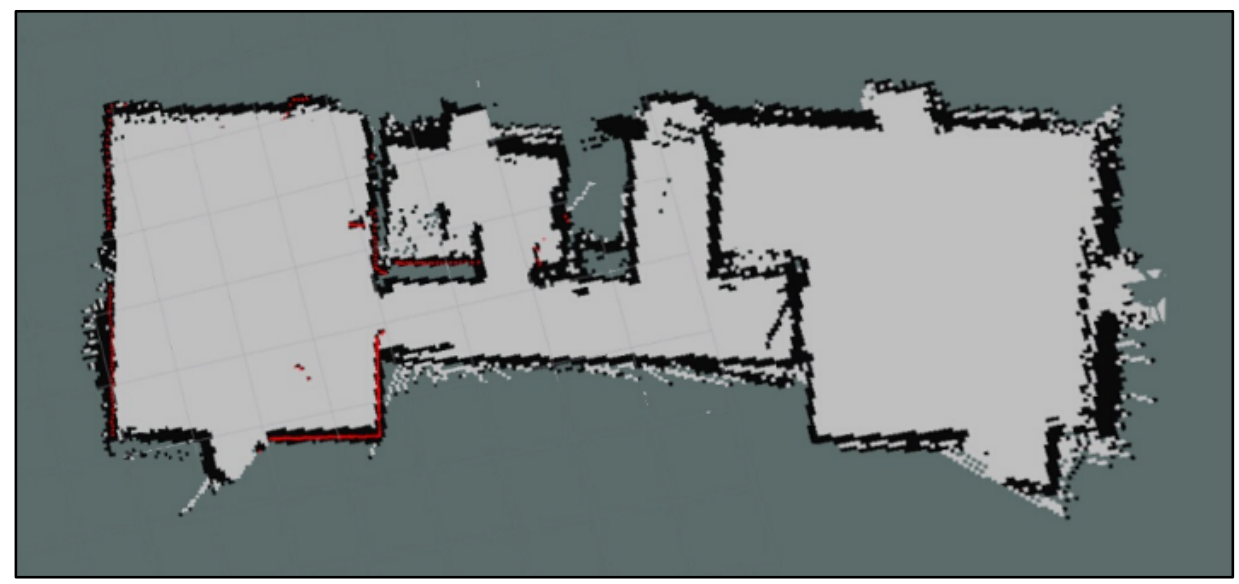

Figure 5. An example map created by SLAM. 


\subsection{Extended Kalman Filter}

The Arducopter can use the Extended Kalman Filter algorithm to estimate the quadcopter's position, velocity and angular acceleration, using signals provided from the gyroscope, accelerometer, magnetometer and other external sensors.

The current version of the ArduPilot primarily uses the EKF algorithm as a source of attitude and position estimation. The algorithm can manage the two separate IMU of the Pixhawk flight controller in parallel.

The EKF can accept external position estimates from the ROS ArUco system.

\subsection{Realtime control}

The system can be controlled remotely via a WiFi network. Connected via SSH (Secure Shell), commands can be sent to the Raspberry Pi single-board computer from the ground control station.

The quadcopter control code must be uploaded to the Raspberry Pi single-board computer prior to take-off, so that any loss of connection to the GCS (ground control station) can even execute the commands smoothly.

The sequence performed during a flight is as follows:

- $\quad$ Launch ArUco pose estimation and Hector SLAM algorithms.

- In case of availability of positional data from external sources, Raspberry Pi establishes connection with the ArduPilot software running on the Pixhawk flight controller via the MAVLink protocol.

- ArduPilot then performs self-testing

- ArduPilot and Raspberry Pi synchronize their clocks. with MAVLink TIMESYNC message.

- $\quad$ Eliminating the lack of GPS signal, the EKF algorithm starts with data from the Marker-based coordinate system

- $\quad$ Running the script with the waypoint commands

The script that issues control commands to the flight controller, sending MAVLink messages on a serial port to the flight controller, first switches the flight mode to Guidedmode, then launches the motors and takes off to a specified height. Then it sends the waypoints as target values to the controller, that navigates along the trajectory. The code is written so that with a remote controller, the user can take the manual control at any time to manually land the quadcopter to avoid any problem.

\section{Experiments}

For testing purposes, markers were placed in a 4 by 4 square pattern at a distance of 1 meter from each other. The camera fixed on the quadcopter has a downward-looking positioning and it defines its position based on the markers as it can be seen in Figure 6. 


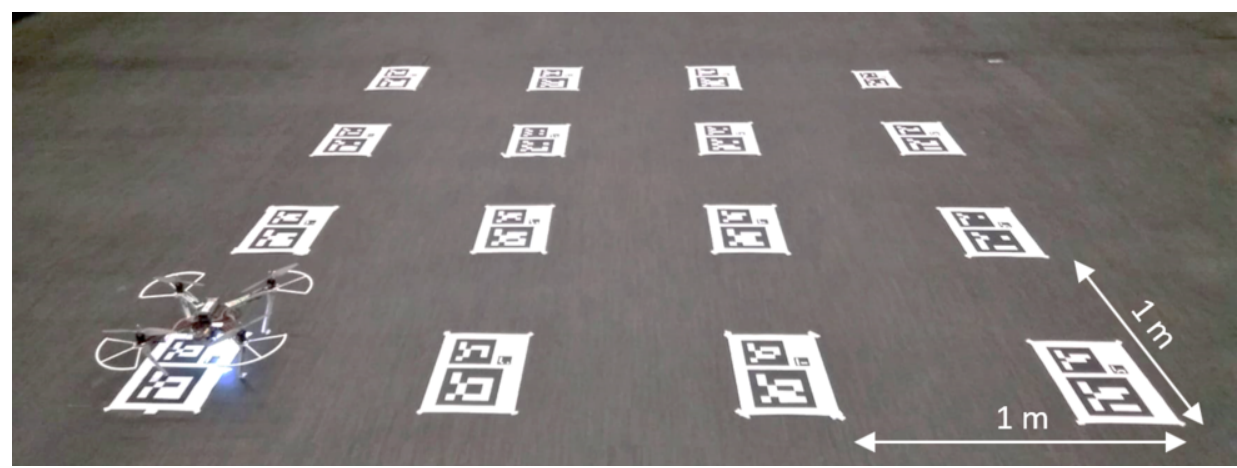

Figure 6. The pattern of the markers.

The precision of the positioning system is tested in two experiments. On the one hand, the data obtained from the onboard camera is compared to the parameters of the Waypoints defined through the program code. The constant recording of the camera data is performed by the rosbag package of the camera topic of the ROS environment, which is a command-line tool. A rosbag package generates a bag-file for data storage. The data recorded in the bag-files are saved with a timestamp, thus assisting the postprocessing of the data. The Robotics System toolbox of the Matlab software allows a detailed analysis of the bag-files.

On the other hand, the movements of the quadcopter are registered through an external system. The measurements were carried out with an OptiTrack MotionCapure system.

The OptiTrack systems measures movement and, through processing the image transmitted from the high-speed infra cameras placed around the measurement area, it can digitally follow the movement of the object with a $0.2 \mathrm{~mm}$ accuracy in a measurement range of $5 \times 4.5 \times 2.2 \mathrm{~m}$.

For the measurement, the ArUco markers were placed 1 to 1 meter apart, and the marker design was arranged according to the previously described arrangement with three different sized markers.

The measurement path contains 4 Waypoint. After the take-off, the quadcopter flies to the Waypoint (1), just above the take-off position, 0.8 meters high, floating in this position, and then starts towards the next Waypoint (2), diagonally above the next marker at a height of 0.8 meters. The Waypoint (3) is below the second waypoint at 0.3 meters. Finally, the quadcopter lands on the marker in the Waypoint (4).

The quadcopter received the following coordinate commands:

$$
\begin{aligned}
& \text { Waypoint (1): } X=0.10 \mathrm{~m}, Y=0.10 \mathrm{~m}, Z=0.80 \mathrm{~m} \\
& \text { Waypoint (2): } X=1.10 \mathrm{~m}, Y=1.10 \mathrm{~m}, Z=0.80 \mathrm{~m} \\
& \text { Waypoint (3): } X=1.10 \mathrm{~m}, Y=1.10 \mathrm{~m}, Z=0.30 \mathrm{~m} \\
& \text { Waypoint (4): } X=1.10 \mathrm{~m}, Y=1.10 \mathrm{~m}, Z=0.00 \mathrm{~m}
\end{aligned}
$$

Along the trajectory defined by the waypoints, in Waypoint 1 and Waypoint 2 the quadcopter has enough height to get 2 different markers in the Field of View at the same 
time, while in Waypoint 3, there is only 1 marker in the Field of View at the height of 0.3 meters.

The following figure 7 shows the representation of one flight separated to coordinates, each numbered area indicates the execution of a given Waypoint command. The dashed line shows the commanded parameters.
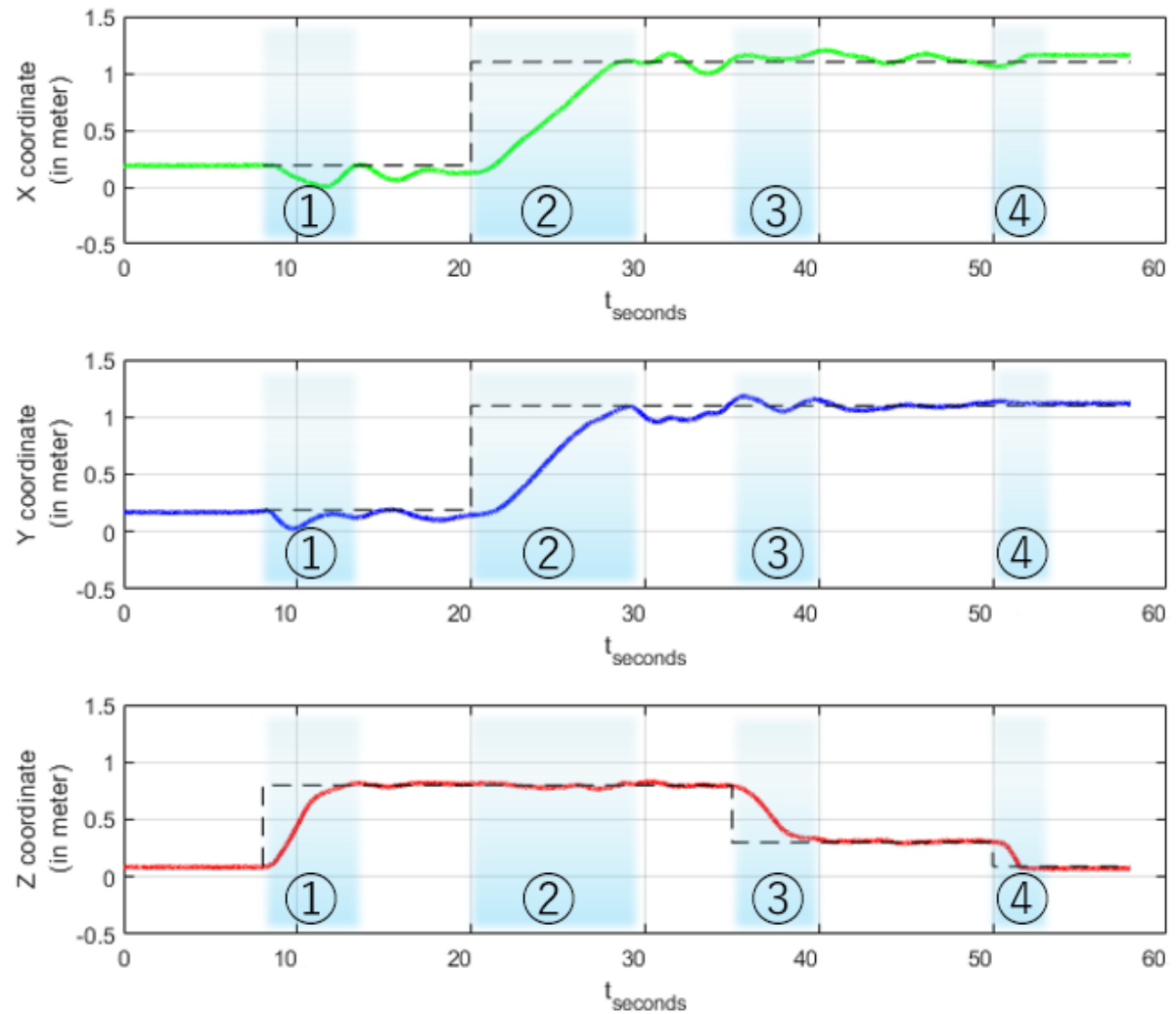

Figure 7. The track measured by an OptiTrack MotionCapure system.

Based on the data from the measurements, marker-based positioning has the error shown in Table 1.

Table 1. The measured error vectors in the waypoints

\begin{tabular}{lcccc}
\hline & Waypoint 1 & Waypoint 2 & Waypoint 3 & Waypoint 4 \\
\hline $\begin{array}{l}\text { Magnitude of mean error } \\
\text { vector }\end{array}$ & $11.5 \mathrm{~mm}$ & $72.1 \mathrm{~mm}$ & $19.3 \mathrm{~mm}$ & $62.6 \mathrm{~mm}$ \\
$\begin{array}{l}\text { Standard deviation of error } \\
\text { vector }\end{array}$ & $30.8 \mathrm{~mm}$ & $70.4 \mathrm{~mm}$ & $43.3 \mathrm{~mm}$ & $42.9 \mathrm{~mm}$ \\
Magnitude of max error vector & $67.9 \mathrm{~mm}$ & $43.3 \mathrm{~mm}$ & $83.2 \mathrm{~mm}$ & $73.6 \mathrm{~mm}$ \\
\hline
\end{tabular}




\section{Conclusion}

The goal of this research was to design a quadcopter and implement a control system with the capability to position the quadcopter indoor using onboard visual pose estimation system, without the help of any GPS.

The research in this paper also covered the design and implementation of quadcopter hardware and software. OpenCV library was used to implement the necessary machine vision. The Open-source-based software was developed in the Robotic Operating System environment, which performs sensor reading and communication with the flight controller while recording data about its operation. To estimate the visual position ArUco marker library for the support of machine vision was implemented in the ROS environment, and LiDAR with Hector SLAM algorithm was used to map the objects around the quadcopter. Computer vision supported positioning system was able to provide a local alternative in the GPS-denied area.

The paper also included the necessary camera calibration. The fusion of positioning data from sensors was achieved by using Extended Kalman Filter.

Finally, with an optical-based external measuring system, the evaluation of the completed positioning system was performed. The introduced evaluation method can be used to investigate the enhancement of positioning performance of UAVs, as well as fine-tuning the parameters of the used controller and filtering approach. As an experience, the right hardware for indoor quadcopter flying is just as important as a decent software solution. It is important to note that the turbulence caused by airflow indoors can reduce system stability.

Sensor-fusion and relying on several physical quantities was proven to be evitable in the enhancement of positioning performance of UAVs. This also highlights the necessity for advancement opportunities.

\section{References}

[1] C. Langlois, S. Tiku, and S. Pasricha, "Indoor Localization with Smartphones: Harnessing the Sensor Suite in Your Pocket," IEEE Consum. Electron. Mag., vol. 6, no. 4, pp. 70-80, 2017, doi: 10.1109/MCE.2017.2714719.

[2] C. N. Trends and D. Industry, "The Drone Industry Barometer 2019,” no. June, 2019.

[3] M. F. Sani and G. Karimian, "Automatic navigation and landing of an indoor AR. Drone quadrotor using ArUco marker and inertial sensors," 1st Int. Conf. Comput. Drone Appl. Ethical Integr. Comput. Drone Technol. Humanit. Sustain. IConDA 2017, vol. 2018-January, no. November 2017, pp. $102-$ 107, 2017, doi: 10.1109/ICONDA.2017.8270408.

[4] W. Sakpere, M. Adeyeye-Oshin, and N. B. W. Mlitwa, "A state-of-the-art survey of indoor positioning and navigation systems and technologies," South African Comput. J., vol. 29, no. 3, pp. 145-197, 2017, doi: 10.18489/sacj.v29i3.452.

[5] A. De Angelis et al., "Design and Characterization of a Portable Ultrasonic Indoor 3-D Positioning System," IEEE Trans. Instrum. Meas., vol. 64, no. 10, pp. 2616-2625, Oct. 2015, doi: 10.1109/TIM.2015.2427892.

[6] R. Mautz, "Indoor Positioning Technologies," Inst. Geod. Photogramm. Dep. Civil, Environ. Geomat. Eng. ETH Zurich, no. February 2012, p. 127, 2012, doi: 10.3929/ethz-a-007313554.

[7] S. Garrido-Jurado, R. Muñoz-Salinas, F. J. Madrid-Cuevas, and M. J. Marín-Jiménez, “Automatic generation and detection of highly reliable fiducial markers under occlusion," Pattern Recognit., vol. 47, no. 6, pp. 2280-2292, 2014, doi: 10.1016/j.patcog.2014.01.005.

[8] M. Nahangi, A. Heins, B. McCabe, and A. Schoellig, "Automated localization of UAVs in GPSdenied indoor construction environments using fiducial markers," ISARC 2018 - 35th Int. Symp. Autom. Robot. Constr. Int. AEC/FM Hackathon Futur. Build. Things, no. Isarc, 2018, doi: $10.22260 /$ isarc $2018 / 0012$. 
[9] V. Mondéjar-Guerra, S. Garrido-Jurado, R. Muñoz-Salinas, M. J. Marín-Jiménez, and R. MedinaCarnicer, "Robust identification of fiducial markers in challenging conditions," Expert Syst. Appl., vol. 93, no. October, pp. 336-345, 2018, doi: 10.1016/j.eswa.2017.10.032.

[10] L. Dowling et al., "Accurate indoor mapping using an autonomous unmanned aerial vehicle (UAV)," 2018.

[11] X. Zuo, P. Geneva, W. Lee, Y. Liu, and G. Huang, "LIC-Fusion: LiDAR-Inertial-Camera Odometry," no. September, 2019.

[12] K. Stefan and S. Oskar, "A Flexible and Scalable SLAM System with Full 3D Motion Estimation," Rapid Commun. Mass Spectrom., vol. 32, no. 5, pp. 407-413, 2011, doi: 10.1002/rcm.8045. 